\title{
Prevalence and subtype of Depression and Anxiety Disorders in referred outpatients from Neurology with headache: Neuro- Psychiatry Interface in Headache
}

\author{
Ashwin Jhalani ${ }^{1}$, Darpan Kaur ${ }^{2}$, Rahul Mishra ${ }^{3}$, Shubhangi Dere ${ }^{4}$, Rakesh Ghildiyal ${ }^{5}$, \\ Kishor Jadhav ${ }^{6}$ \\ ${ }^{1}$ Resident, Department of Psychiatry \\ ${ }^{2}$ Associate Professor, Department of Psychiatry \\ ${ }^{3}$ Senior Resident, Department of Psychiatry \\ ${ }^{4}$ Assistant Professor, Department of Psychiatry \\ ${ }^{5}$ Professor \& Head of Department, Department of Psychiatry \\ ${ }^{6}$ Assistant Professor, Department of Neurology \\ Mahatma Gandhi Mission Medical College, Navi Mumbai \\ Corresponding author: Ashwin Jhalani \\ Email-drashwinjhalani@yahoo.com
}

\begin{abstract}
Background and Objectives: Headache is an extremely common symptom and collectively headache disorders are among the most common of the nervous system disorders. Primary headache disorders migraine, tension headache and cluster headache - constitute nearly $98 \%$ of all headaches; however, secondary headaches are important to recognize as they are serious and may be life threatening. The aim of this study is to study the prevalence of depression, anxiety and subtypes of depression and anxiety with headaches, using pertinent clinical test profiles.

Methods: A cross sectional survey conducted to study depression and anxiety symptoms in patients with headache presenting in neurology OPD with study of MRI findings and associated medical co morbidities. Data was collected over a period of 2 months and study setting was at MGM Medical College, Navi Mumbai.

Results: $32 \%$ reported no depressive features, while $36 \%$ reported features suggestive of mild depression, $18 \%$ had moderate depression, $8 \%$ had severe depression and $6 \%$ reported to have very severe depression. $60 \%$ of the patients reported to have mild anxiety while $22 \%$ had mild to moderate anxiety and $18 \%$ had moderate to severe anxiety.

Conclusion: Depressive disorders are very common comorbid psychiatric disorders among the patients presenting with headache followed by anxiety disorders. Female gender is more likely to suffer from an underlying Depressive or Anxiety disorder. Tension type headache is one of the most common presentation in majority of these patients. Familial stressors, adjustment problems, marital disharmony and financial stressors add to the occurrence of Headache as recurring symptom as it was seen in our study.
\end{abstract}

Keywords: Depression, anxiety, subtype, headache.

(Paper received $-9^{\text {th }}$ September 2018, Peer review completed $-20^{\text {th }}$ October 2018)

(Accepted $-24^{\text {th }}$ October 2018)

\section{INTRODUCTION}

Headache is an extremely common symptom and collectively headache disorders are among the most common of the nervous system disorders, with a prevalence of $48.9 \%$ in the general population. Headache affects people of all ages, races and socioeconomic status and is more common in women. Some headaches are extremely debilitating and have significant impact on an individual's quality of life. Primary headache 
disorders - migraine, tension headache and cluster headache - constitute nearly $98 \%$ of all headaches; however, secondary headaches are important to recognise as they are serious and may be life threatening. [1]. There has been much discussion about a possible relationship between psychological factors and headache for a very long time. In 1937, Wolff defined the "migraine personality" including ambition and perfectionism, mental instability and immaturity, vulnerability to frustrations, and shyness. The psychiatric diagnoses most commonly associated with headaches are anxiety and depressive disorders and the personality trait most frequently associated is neuroticism. Population-based studies have confirmed this positive association in patient samples. In the Zurich Cohort Study of Young Adults, the combination of anxiety disorder and major depression was significantly associated with migraine [2]. Literature data show that psychiatric comorbidity in migraine and tension-type headache is very similar ( $88 \%$ and $85 \%$ respectively). Anxiety disorders is the most frequent association (54.9\% and 52.5\% respectively) followed by mood disorders (34.4\% and $36.4 \%$ respectively). Edward Leiving described the psychiatric symptoms of migraine, depressed mood, irritability, anxiety, fatigue and impaired memory concentration over 100 years ago. Although this pioneer observation has been confirmed in successive studies conducted over decades in both psychiatric and neurological settings, it is only recently that the psychiatric comorbidity with primary headache has been systematically examined [3]. There are good reasons for wishing to establish whether or not there are associations between the common headache disorders and the common psychiatric disorders, all of them major contributors to public ill health and the burden of disability [4]. The aim of the current study was to study prevalence of Depressive disorders and Anxiety in patients with headache and to assess subtypes of depression and anxiety disorders in this group.

\section{METHODOLOGY}

It was a cross sectional survey conducted to study depression and anxiety symptoms in patients with headache presenting in neurology OPD with study of MRI findings and associated medical co morbidities. Data was collected over a period of 2 months and study setting was at MGM Medical College, Navi Mumbai. All patients attending the neurology clinic presenting with complaints of headache MGM Hospital, Kamothe, Navi Mumbai were eligible to participate in the study. The inclusion criteria comprised of cases who presented with a complaint of headache were clinically interviewed by the Consultant and were given appropriate clinical diagnosis and provided standard psychiatric rating scale to assess depression and anxiety. Patients willing to participate in the study after filling an informed consent were included in the study. The data was collected using a semi structured proforma. Data was analysed for demographic factors, psychiatric diagnosis, neurological diagnosis, medical co morbidities and MRI findings if done were recorded. Data was entered in excel sheet and Data collected was analysed using SPSS 20. Institutional Ethics clearance was obtained.

\section{Scales Used -}

Hamilton Anxiety Rating Scale (HAM -A): The Hamilton Anxiety Rating Scale (HAM-A, sometimes termed HARS), dating back to 1959 , is one of the first rating scales to measure the severity of perceived anxiety symptoms. It is still in use today, being considered one of the most widely used rating scales, and has been translated into Cantonese, French and Spanish. It has been used as a benchmark for more recently devised scales, It consists of 14 symptom-defined elements, and caters for both psychological and somatic symptoms, comprising anxious mood; tension (including startle response, fatigability, restlessness); fears (including of the dark/strangers/crowds); insomnia; 'intellectual' (poor memory/difficulty concentrating); depressed mood (including anhedonia); somatic symptoms (including aches and pains, stiffness, bruxism); sensory (including tinnitus, blurred vision); cardiovascular (including tachycardia and palpitations); respiratory (chest tightness, choking); gastrointestinal (including irritable bowel syndrome-type symptoms); genitourinary (including urinary frequency, loss of libido); autonomic (including dry mouth, tension headache) and observed behaviour at interview (restless, fidgety, etc.). Each item is scored on a basic numeric scoring of 0 (not present) to 4 (severe): $>17 / 56$ is taken to indicate mild anxiety; $25-30$ is considered moderate-severe [5]. 
Hamilton Depression Rating Scale (HAM-D): The Hamilton Rating Scale for Depression (HAM-D) is one of the longest standing, most widely used measures of depression severity in research and clinical practice. Originally designed to measure symptom severity in depressed inpatients, the 17-item HAM-D has evolved over the past 50 plus years into 11 modified versions that have been administered to various patient populations in an array of psychiatric, medical, and other research settings. Bagby and colleagues examined internal reliability using Chronbach's alpha. They found alphas ranging from $0.46-0.92$. In eight of the 12 studies reporting Chronbach's alphas, internal reliability coefficients were less than or equal to 0.76 [6].

\section{Inclusion Criteria:}

1. Patients between 18 to 60 years of age.

2. Patients willing to participate in the study.

\section{Exclusion Criteria:}

Patients having severe psychiatric and severe medical illness, mental retardation or sensory impairments rendering them uncooperative for assessments and consent shall be excluded from the study.

\section{RESULTS}

\section{Demographic Profile}

Study comprised of 50 individuals who were referred from Neurology clinic to psychiatry with consistent complaints of headache and had been treated earlier without a desirable outcome. These patients were assessed using HAM-A and HAM-D scale for subsequent assessment for anxiety and depression. Of all the participants majority of the $29(58 \%)$ were women and $21(42 \%)$ were male.

\section{HAM-D Profile}

HAM-D profile consisted of assessing the levels of depression in 5 categories consisting of Normal or no depression, Mild depression, Moderate depression, Severe depression, Very severe depression. 16 (32\%) reported no depressive features, while $18(36 \%)$ reported features suggestive of mild depression, $9(18 \%)$ had moderate depression, $4(8 \%)$ had severe depression and $3(6 \%)$ reported to have very severe depression.

\section{HAM-A Profile}

HAM-A profile results were classified as mild anxiety, mild to moderate anxiety, and moderate to severe anxiety. $30(60 \%)$ of the patients reported to have mild anxiety while $11(22 \%)$ had mild to moderate anxiety and $9(18 \%)$ had moderate to severe anxiety.

\section{Psychiatric Illness Profile}

Of the 50 respondents 12 (24\%) patients with complaints of headache had Diagnosis of Major Depressive disorder, $12(24 \%)$ had diagnosis of Generalized Anxiety Disorder, 8 (16\%) of the patients had Somatoform Disorder, 5 (10\%) had adjustment disorder, $4(8 \%)$ had Panic Disorder, another $4(8 \%)$ were given a diagnosis of Mixed Anxiety Depression, 2 (4\%) of the respondents had a diagnosis of Social anxiety disorder, $2(4 \%)$ had diagnosis of migraine and $1(2 \%)$ of the total patients had Dysthymia.

\section{DISCUSSION}

Researchers in their study on depression increasing tension type headache found that almost all the participants with headache had association with depression and who were not likely diagnosed otherwise also Depression increased the risk of headache and the individuals in the headache prone/depressed group reported experiencing a headache [7]. In our study almost $68 \%$ of the respondents had Depression depicted on HAM D ranging from mild to very severe depression.

Other researchers in their study found co morbid Depression in almost (48.5\%) patients with headache while (17.90\%) suffered from anxiety related disorders (generalized anxiety disorder or agoraphobia or social phobia or panic disorder). In our study we found depression to be co morbid in $68 \%$ of the patients with Major Depressive Disorder been the most common diagnosis and Generalized anxiety disorder to be most 
common anxiety disorder while $60 \%$ of the patients had Mild anxiety $60 \%, 22 \%$ had moderate to severe anxiety and $18 \%$ had severe anxiety in our study [8].

In yet another study, it was found that Depression and anxiety were prevalent both amongst men and women with ration of 28:64, we found that ratio to be $58: 42$, the study indicates that majority of women above the age group of 40 and above presenting with complaints of headache and body ache are mostly having comorbid depression or anxiety in our we found patients having co morbid Depression or Anxiety or both in majority of the patients [9].

In study on the Prevalence of psychiatric co-morbidity in patients presenting with chronic daily headache: a hospital based cross sectional study found psychiatric disorders to be co morbid with patients presenting with complaints of headache in $74 \%$ of the patients [10]. Out of $74 \%$ of the psychiatric illnesses; $28 \%$ were suffering from affective spectrum disorders, $16 \%$ were suffering from anxiety disorders, $16 \%$ had psychotic disorders, $12 \%$ had other neurotic stress related disorders ( $6 \%$ had adjustment disorder, $5 \%$ had dissociative disorders, and $1 \%$ had somatization disorder) and $2 \%$ had personality disorder. While the ambit of out study was limited we did find the psychiatric morbidity to be quiet high amongst the patients and $24 \%$ of them had Major Depressive Disorder, 24\% had Generalized anxiety Disorder, $16 \%$ of the patients has Somatoform Disorder , 10\% had Adjustment disorder, $8 \%$ had Panic disorder, $8 \%$ had Mixed anxiety Depression $4 \%$ patients had co morbid Social anxiety disorder, while another $4 \%$ had migraine as their main diagnosis, while $2 \%$ of the patients had Dysthymia [10].

\section{CONCLUSION}

Depressive disorders are very common comorbid psychiatric disorders among the patients presenting with headache followed by anxiety disorders. Female gender is more likely to suffer from an underlying Depressive or Anxiety disorder. Tension type headache is one of the most common presentation in majority of these patients. Taboo against psychiatrist and lack of psychiatrist throughout India means that majority of these patients are being treated by either Neurologists or General Physicians who may not be trained in Psychiatry and are likely to diagnose majority of cases as migraine, and patients being not relieved of their symptoms for log duration until eventually they get referred to a psychiatrist. Familial stressors, adjustment problems, marital disharmony and financial stressors add to the occurrence of Headache as recurring symptom as it was seen in our study.

\section{Limitations of the Study}

A relatively small sample size and also selective intake of patients as these patients were refereed from Neurology OPD having undergone treatment prior to being referred to psychiatry adds to the high prevalence of psychiatric morbidity. Most of the patients referred had a co morbid psychiatric illness. Other parameters were left out of the study that could have been included that of socioeconomic data and its likely impact could have been studied, educational profile could have been studied. A follow up study of these patients undergoing psychiatric treatment and re assessment could have predicted the better efficacy of patients with headache been treated with psychiatric medications.

\section{REFERENCES}

1. Ahmed F. Headache disorders: differentiating and managing the common subtypes. Br J Pain 2012;6(3):12432 .

2. Bensenor IM, Tofoli LF, Andrade L. Headache complaints associated with psychiatric comorbidity in a population-based sample. Braz J Med Biol Res 2003;36(10):1425-32.

3. Palagini L, Balsamo EL, Panicucci P, Guazzelli M. Primary headache and comorbidity with mood and anxiety disorders: an example of the psycho-neuro-biological loop. J Headache Pain 2000;1(1):S17-9.

4. Lampl C, Thomas H, Tassorelli C, Katsarava Z, Laínez JM, Lantéri-Minet M, Rastenyte D, de la Torre ER, Stovner LJ, Andrée C, Steiner TJ. Headache, depression and anxiety: associations in the Eurolight project. J Headache Pain 2016;17(1):59-66.

5. Thompson E. Hamilton rating scale for anxiety (HAM-A). Occup Med 2015;65(7):601-9. 
6. Rohan KJ, Rough JN, Evans M, Ho SY, Meyerhoff J, Roberts LM, Vacek PM. A protocol for the Hamilton Rating Scale for Depression: item scoring rules, rater training, and outcome accuracy with data on its application in a clinical trial. J Affect Disord 2016;200:111-8.

7. Janke EA, Holroyd KA, Romanek K. Depression increases onset of tension-type headache following laboratory stress. Pain 2004;111(3):230-8.

8. Desai SD, Pandya RH. Study of psychiatric comorbidity in patients with headache using a short structured clinical interview in a rural neurology clinic in Western India. J Neurosci Rural Pract 2014;5(Suppl 1):S39-45.

9. Singh AK, Shukla R, Trivedi JK, Singh D. Association of psychiatric co-morbidity and efficacy of treatment in chronic daily headache in Indian population. J Neurosci Rural Pract 2013;4(2):132-6.

10. Bhuvana RC. Prevalence of psychiatric co-morbidity in patients presenting with chronic daily headache: a hospital based cross sectional study. Int J Res Med Sci 2016;5(1):321-5.

\section{Acknowledgements - Nil \\ Conflict of Interest - Nil \\ Funding - Nil}

